\title{
Infectious Agents and Cancer reviewer acknowledgement 2015
}

Franco M. Buonaguro ${ }^{1 *}$, Sam M. Mbulaiteye ${ }^{2}$ and Maria Lina Tornesello ${ }^{3}$

\section{Contributing reviewers}

The editors of Infectious Agents and Cancer would like to thank all our reviewers who have contributed their time and expertise to the journal in Volume 10 (2015).

Sayed Abdelwahab

Egypt

Ali Abdualsalam

Yemen

Amit Achhra

Australia

Clement Adebamovo

USA

Francisco Aguayo

Chile

Kayode Ajenifuja

Nigeria

Vidya Ajila

India

Elena Allia

Italy

Clorinda Annunziata

Italy

Ines Badano

Argentina

Iacopo Baussano

France

Robert Biggar

USA
Sabrina Bimonte

Italy

Julia Bohlius

Switzerland

Patrizia Bonelli

Italy

Álvaro H Borges

Denmark

Idris Brima

Sudan

Franco Buonaguro

Italy

Monica Cantile

Italy

Francesca Carozzi

Italy

Flair J Carrilho

Brazil

Sekar Pedamallu Chandra

USA

Kimon Chatzistamatiou

Greece

Carla Chibwesha

South Africa
Admire Chikandiw

South Africa

Lucy Chimoyi

South Africa

Nyasha Chin'Ombe

Zimbabwe

Roberto Cianni

Italy

Anna Coghil

USA

Manola Comar

Italy

Gaetano Corazzelli

Italy

Silvia De Sanjose

Spain

Annarosa Del Mistro

Germany

Katarzyna Derwich

Poland

Paola Di Bonito

Italy

Juan Du

United States

\footnotetext{
* Correspondence: irccsvir@unina.it

${ }^{1}$ Istituto Nazionale Tumori, Naples, Italy

${ }^{2}$ National Cancer Institute, Bethesda, USA

${ }^{3}$ Istituto Nazionale Tumori, Naples, Italy
} 
Ola Forslund

Sweden

Renato Franco

Italy

Aharon Freud

USA

E. M. Fuentes-Panama

Mexico

Franco Fulciniti

Switzerland

Marisa Gariglio
Italy

Italy

Paolo Giorgi Rossi

Italy

Lucia Giovannelli

Italy

Paolo Giovenali

Italy

Patricia Goggin

Canada

Shalaka Hampras

USA

Maria Hatziapostolou

USA

Andrew M Hill

UK

Dieter Hoffmann

Germany

Jin Huang

China

Tetsuya Ikeda

Japan

Somayeh Jalilvand

Iran

Phyllis J Kanki

USA

Shyam Kottilil

USA

Mineo Kurokawa

Japan

Anton Kutikhin

UK

Ke Lan

China
Wen Li

China

Xingnan Li

USA

Flavia Lillo

Italy

Morgan Marks

USA

Koji Matsumoto

Japan

Sam Mbulaiteye

USA

Alicia Mcdonald

USA

L Menezes

Brazil

J Mosmann

Argentina

Emmanuel Mugisha

Uganda

P G Murray

UK

Jonah Musa

Nigeria

Luciano Mutti

UK

Miriam Nakalembe

Uganda

Jacques Neefjes

Netherlands

Twalib Ngoma

Tanzania

Souichi Nukuzuma

Japan

Josè Oteo

Spain

Ridha Oueslati

Tunisia

Giuseppe Palma

Italy

H R Park

South Korea

Francesca Pentimalli

Italy
Annacarmen Petrizzo

Italy

Francesca Pezzuto

Italy

Eric Piaton

France

Maurizio Provenzano

Italy

Fabiana Quaglia

Italy

Camille Ragin

USA

Yasir Raza

Pakistan

Matejka Rebolj

Denmark

Milan Reinis

Czech Republic

Chad Ritch

USA

Frank Romeo

Italy

Salvatore Ronsini

Italy

Giovanni Battista Rossi

Italy

Martha-Eugenia Ruiz-Tachiquin Mexico

Adolfo G. Sadile

Italy

Pierre Saintigny

France

Martina Salakova

Czech Republic

Mauricio Salcedo

Mexico

Omer Salim

Sudan

Sergio Sandrucci

Italy

Rodrigo Silvestre Brazil

Kenneth Simbiri

USA 
Freddy Sitas

Australia

Leon Snyman

South Africa

Nema Soliman

Egypt

Cheng Song

China

Madeleine Sporleder

Germany

Jatupol Srisomboon

Thailand

Noemy Starita

Italy

Giovanni Stellato

Italy

Caecilia Hapsari Ceriapuri Sukowati Italy
Martins Tanimola

UK

Mauro Tognon

Italy

Maria Lina Tornesello

Italy

Ines Tozetti

Brazil

M Trimech

France

Piamkamon Vacharotayangul

Thailand

Hernán Vargas

Colombia

Aldo Venuti

Italy

Fernanda Visioli

Brazil
Monika Wagner

Canada

Thomas Weiss

USA

Denise Whitby

USA

$\mathrm{Xin} \mathrm{Xu}$

USA

Geng Yu

USA

Sonia Zapata

Ecuador

Apostolos Zaravinos

Sweden

Huachun Zou

Australia

Antonella Zucchetto

Italy 